\title{
The Dopamine Transporter Is Localized to Dendritic and Axonal Plasma Membranes of Nigrostriatal Dopaminergic Neurons
}

\author{
Melissa J. Nirenberg, ${ }^{1}$ Roxanne A. Vaughan, ${ }^{2}$ George R. Uhl, ${ }^{3,4,7}$ Michael J. Kuhar, ${ }^{2,5,6,7}$ and \\ Virginia M. Pickel ${ }^{1}$ \\ 1 Dopartment of Nourology and Nouroscionce, Cornoll University Medical College, New York, New York 10021. \\ ${ }^{2}$ Neuroscience and 3 Molecular Neurobiology Branches, National Institute on Drug Abuse, Baltimore, Maryland 21224, \\ and Departments of ${ }^{4}$ Neurology, ${ }^{5 P h a r m a c o l o g y,}{ }^{6}$ Psychiatry, and 7 Neuroscience, The Johns Hopkins University School \\ of Medicine, Baltimore, Maryland 21224
}

Nigrostriatal dopaminergic neurons play an essential role in the central regulation of motor functions. These functions are initiated through the release of dopamine from axon terminals in the striatum or from dendrites in the substantia nigra (SN) and are terminated by the reuptake of dopamine by the sodiumand chloride-dependent dopamine transporter (DAT). DAT also can transport dopamine neurotoxins and has been implicated in the selective vulnerability of nigrostriatal dopaminergic neurons in major models of Parkinson's disease. We have used electron microscopic immunocytochemistry with an $\mathrm{N}$-terminal domain anti-peptide antibody to examine the subcellular distribution of DAT in the rat SN and dorsolateral striatum. In the SN, immunogold labeling for DAT was localized to cytoplasmic surfaces of plasma membranes and smooth endoplasmic reticulum of dendrites and dendritic spines, few of which contained synaptic vesicles. Neuronal perikarya in the SN contained immunogold-labeled pleomorphic electron-lucent tubulovesicles but showed immunolabeling of plasma membranes only rarely. Axon terminals in the striatum contained extensive immunogold labeling of cytoplasmic surfaces of plasma membranes near aggregates of synaptic vesicles and less frequent labeling of intervaricose segments of plasma membrane or small electron-lucent vesicles. In sections dually labeled for DAT and the catecholamine-synthesizing enzyme tyrosine hydroxylase, both markers were colocalized in most profiles in the SN and striatum. These findings support the proposed topological model for DAT and suggest that this transporter is strategically located to facilitate uptake of dopamine and neurotoxins into distal dendritic and axonal processes of nigrostriatal dopaminergic neurons.

Key Words: dopamine transporter; dopamine reuptake; im munogold; substantia nigra; striatum; caudate-putamen; neurotoxicity; Parkinson's disease; dendritic release; reverse transport; amphetamine; cocaine
Nigrostriatal dopaminergic neurons are critically important in the central regulation of motor functions (for review, see Björklund and Lindvall, 1984; Roth and Elsworth, 1995). In these neurons, transmission is initiated by the regulated release of dopamine from presynaptic axon terminals in the striatum and/or from presynaptic dendrites in the substantia nigra (SN) (Cheramy et al., 1981; Nissbrandt et al., 1989). After it is released into the extracellular space, dopamine interacts with receptors located on the plasma membranes of ncurons or glial cells (Seeman, 1995). The termination of dopaminergic transmission is also highly regulated and is caused primarily by the active reduction of extracellular dopamine and chloride concentrations by a sodium- and chloridedependent dopamine uptake carrier (Roth and Elsworth, 1995). Under certain conditions in which the concentration gradients are reversed, this dopamine carrier also can mediate the calciumindependent release of dopamine and coupled ions via reversal of

Received July 11, 1995; revised Oct. 5, 1995; accepted Oct. 9, 1995.

M.J.N. was supported in part by National Institute of General Medical Sciences Grant GM07739. V.M.P. was supported by National Institute of Mental Health Grants MH00078 and MH40342 and National Institute on Drug Abuse Grant DA04600. R.A.V., G.R.U., and M.J.K. were supported by the Intramural Research Program, National Institute on Drug Abuse. We thank Dr. Adena L. Svingos for critical commentary and Terence M. Ziegler for photographic assistance.

Correspondence should be addressed to Melissa J. Nirenberg, Department of Neurnlngy and Neuroscience, Cornell University Medical College, 411 East 69th Street, Room KB 410, New York, NY 10021.

Copyright (C) 1996 Society for Neuroscience $0270-6474 / 96 / 160436-12 \$ 05.00 / 0$ the normal direction of transport (Ratieri et al., 1979; Sulzer et al., 1993; Bannon et al., 1995).

Molecular cloning techniques recently have identified a single gene encoding a dopamine transporter (DAT) that confers all of the pharmacological properties of the rat dopamine reuptake carrier (Giros et al., 1991; Kilty et al., 1991; Shimada et al., 1991), including the capacity to bind cocaine and other psychostimulant drugs (Ritz et al., 1987; Kuhar et al., 1991). The highest levels of DAT mRNA are expressed in midbrain dopaminergic neurons, particularly those in the ventromedial SN pars compacta (SNC) (Shimada et al., 1992; Cerruti et al., 1993). Studies of radioligand binding to dopamine uptake sites (Graybiel and Moratalla, 1989) and, more recently, light microscopic immunocytochemistry for DAT (Ciliax et al., 1995; Freed et al., 1995; Revay et al., in press) have shown that DAT is expressed at the highest levels in the patch compartments of the striatum, which are known to receive dense inputs from dopaminergic perikarya in the ventral tier of the SNC (Gerfen et al., 1987). Interestingly, these nigrostriatal dopaminergic neurons are also the most susceptible both to exogenous neurotoxins and to specific neurodegenerative diseases (Manaye et al., 1989; Kaakkola and Teräväinen, 1990; Pickel et al., 1992). Because DAT can transport dopamine neurotoxins, the expression of high levels of DAT in nigrostriatal dopaminergic neurons represents an attractive candidate model to explain this selective vulnerability (Javitch et al., 1985; Kitayama et al., 1992; Edwards, 1993). 
Although the importance of DAT in regulating dopaminergic transmission has been clearly established, the precise subcellular localization of this transporter is not yet known. The recent availability of high-titer polyclonal antisera against rat DAT (Freed et al., 1995) has enabled us to use electron microscopic immunocylochemistry to examine the subcellular localization of DAT in nigrostriatal dopaminergic neurons. To confirm that the labeled neurons are dopaminergic, we have double-labeled some of the sections for both DAT and the catecholamine-synthesizing enzyme tyrosine hydroxylase (TH). The results identify the potential sites of synthesis, transport, and functional expression of DAT in rat nigrostriatal dopaminergic neurons.

\section{MATERIALS AND METHODS}

Antisera. The DAT antiserum that was used in these experiments is a high-titer rabbit polyclonal antiserum directed against a synthetic peptide (LTNSTLINPPQTPVEAQE), "peptide 16," corresponding to amino acids 42-59 near the $\mathrm{N}$ terminus of DAT (Freed et al., 1995). The specificity of this antiserum has been established previously by enzyme-linked immunosorbent assay, Western analysis, and immunoprecipitation studies (Freed et al, 1995). The TH antiserum that was used in these experiments was a commercially available mouse monoclonal antiserum directed against $\mathrm{TH}$ (Incstar, Stillwater, $\mathrm{MN}$ ).

Tissue preparation. The methods for tissue preparation and immunolabeling were based on those described by Leranth and Pickel (1989). Twelve adult male Sprague-Dawley rats $(250-400 \mathrm{gm})$ were anesthetized with sodium pentobarbital ( $50 \mathrm{mg} / \mathrm{kg}$, i.p.). They were then perfused through the ascending aorta with $40 \mathrm{ml}$ of heparin $(1000 \mathrm{U} / \mathrm{ml}$ heparin in $0.15 \mathrm{M} \mathrm{NaCl}$, $50 \mathrm{ml}$ of $3.75 \%$ acrolein, and $200 \mathrm{ml}$ of $2 \%$ paraformaldehyde in $0.1 \mathrm{M}$ phosphate buffer ( $\mathrm{PB} ; \mathrm{pH} \mathrm{7.4)}$. The brains were removed and post-fixed for $30 \mathrm{~min}$ in $2 \%$ paraformaldehyde. Sections through the $\mathrm{SN}$ and striatum were cut on a Lancer vibratome at a thickness of $30-40$ $\mu \mathrm{m}$, incubated for $30 \mathrm{~min}$ in a solution of $1 \%$ sodium borohydride in PB to remove active aldehydes, and rinsed in $\mathrm{PB}$ until bubbles no longer emerged from the tissue. They were then cryoprotected for $15 \mathrm{~min}$ in a solution of $25 \%$ sucrose and $3.5 \%$ glycerol in $0.05 \mathrm{M} \mathrm{PB}$, rapidly frozen in chlorodifluoromethane followed by liquid nitrogen, and thawed in PB at room tempcraturc.

Immunocytochemistry. All incubations were carried out at room temperature with agitation and were followed by several washes with $\mathrm{PB}$,

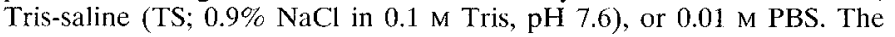
sections were incubated in one of the following primary antibody solutions, all of which were prepared in $0.1 \%$ bovine serum albumin (BSA)TS: (1) anti-DAT 1:6000 for single labeling; (2) anti-DAT 1:6000 and anti-TH 1:10,000 for double labeling; or (3) anti-DAT 1:10,000 and anti-TH 1:2000 for double labeling with reversed markers. In addition, the following antisera were prepared in $0.1 \%$ BSA-TS and incubated for 18-24 hr at room temperature before use: (1) $1 \mathrm{ml}$ of anti-DAT 1:1000 and $1-2 \mathrm{mg} / \mathrm{ml}$ peptide 16 as a negative (adsorption) control; and (2) $1 \mathrm{ml}$ of anti-DAT 1:1000 and 1-2 $\mathrm{mg} / \mathrm{ml}$ unrelated DAT peptide, "peptide 12 " (Freed et al., 1995), as a positive control. Before use, these negative and positive control antisera were diluted with $5 \mathrm{ml}$ of $0.1 \%$ BSA-TS to final primary antibody concentrations of 1:6000.

For silver-enhanced immunogold labeling (Chan et al., 1990), the bound DAT or TH antibodies were identified as follows. The sections were incubated for 2 hr in a 1:50 dilution of colloidal gold (1 nm)-labeled anti-rabbit (for DAT) or anti-mouse (for TH) immunoglobulin (Ig) G (Amersham, Arlington Heights, IL), fixed for 10 min in $2 \%$ glutaraldehyde in PBS, and reacted for $3-7 \mathrm{~min}$ (for electron microscopy) or 10-14 $\mathrm{min}$ (for light microscopy) with a silver solution using a light-stable IntenSEM kit (Amersham). For immunoperoxidase labeling, the bound primary DAT antibodies were labeled using the avidin-biotin complex method (Hsu et al., 1981) as follows. Sections were incubated for $30 \mathrm{~min}$ in a 1:400 dilution of biotinylated goat anti-rabbit IgG in $0.1 \% \mathrm{BSA}$, for $30 \mathrm{~min}$ in a $1: 100$ dilution of avidinbiotin-peroxidase complex, and then for $6 \mathrm{~min}$ in a solution consisting of 22 $\mathrm{mg}$ of $3,3^{\prime}$-diaminobenzidine (DAB) and $10 \mu \mathrm{l}$ of $30 \% \mathrm{II}_{2} \mathrm{O}_{2}$ in $100 \mathrm{ml}$ of 0.1 $\mathrm{M}$ TS, pH 7.6. For double-immunolabeling, the immunoperoxidase reaction for TH (or DAT) was followed by immunogold-silver detection for the other antigen. Sections prepared for light microscopic examination were rinsed several times in PB, mounted on glass slides, and dried overnight in a desiccator. They were then dehydrated, coverslipped with Permount, and examined on a Nikon microscope using differential interference contrast optics.
Electron microscopy. Sections prepared for electron microscopy were fixed in $2 \%$ osmium tetroxide for $60 \mathrm{~min}$, dehydrated in a series of graded ethanols and propylene oxide, and flat-embedded in Epon 812 between two pieces of Aclar plastic. Ultrathin sections were collected from the outer surface of the plastic-embedded tissue using an RMC ultramicrotome (Research and Manufacturing Company, Tuscon, AZ). Sections from the SN were sampled from the medial third of the pars compacta and pars reticulata. Striatal sections were sampled from the dorsolateral portion of the caudate-putamen nucleus just ventral to the corpus callosum, at rostrocaudal levels in which the crossing of the anterior commissure also was observed. All of the ultrathin sections then were counterstained with lead citrate and uranyl acetate and examined with a Philips 201 elcctron microscope (Mahwah, NJ).

Structures were considered to be immunogold-labeled when they were in direct contact with at least one gold particle and contained within a profile in which there were at least two gold particles. Such small numbers of gold particles were considered to denote specific labeling because of the extremely low background of the primary antiserum and its highly selective regional and subcellular localization. Because these experiments localized DAT using a single anti-peptide antiserum, however, we cannot rule out the possibility that the absence of labeling in a particular portion of the plasma membrane or a particular subcellular organelle is attributable to loss or masking of the N-terminal domain of DAT, against which the antiserum was directed. For semiquantitative analysis, the maximal cross-sectional diameters in the observed plane of section were measured for 174 dendrites containing immunogold or immunoperoxidase labeling for DAT. The number of unlabeled terminals in direct contact with each of these DAT-labeled dendrites was tabulated and reported as the mean number of contacts $\pm \mathrm{SD}$.

\section{RESULTS}

Consistent patterns of immunoperoxidase and immunogold labeling for DAT were seen within dendrites (Figs. 1-3) and perikarya (Fig. 4) in the SN, and within axons (Fig. 5A,B) and axon terminals (Figs. $5 C, D, 6,7$ ) in the dorsolateral striatum. No detectable labeling of glial profiles or other non-neural elements was observed in either region. As in previous experiments (Freed et al., 1995), the immunolabeling was eliminated by preadsorption of the primary antiserum with the peptide against which the antiserum was generated but was unaffected by preadsorption with an unrelated DAT peptide. Immunolabeling for DAT was seen in sections that were sampled for electron microscopy from the surface of the tissue (Figs. 1-7) but was absent from sections sampled from deeper within the tissue, where immunoreagent penetration was poor.

\section{DAT localizes to the plasma membranes and smooth endoplasmic reticulum of dendrites in the SN}

In the SNC and SN pars reticulata (SNR), DAT immunolabeling was observed most often in dendrites. Most $(65 \%)$ of these DAT-immunoreactive dendrites were $<5 \mu \mathrm{m}$ in maximal crosssectional diameter in the observed plane of section (Fig. 1). The labeled dendrites were frequently contacted by one or more (2.7 \pm 1.7 ) unlabeled axon terminals (Fig. 1). These axon terminals sometimes formed symmetric or asymmetric synaptic junctions onto the labeled dendrites, but often these junctions could not be resolved clearly (Fig. 1).

Subcellular examination of the labeled dendrites showed that numerous immunogold particles for DAT were localized to cytoplasmic surfaces of plasma membranes (Figs. 1, 2). In some cases, these labeled segments of plasma membrane were apposed to (Fig. 1) or received synaptic junctions from unlabeled axon terminals (Fig. 1 $A, C$ ). The immunogold particles for DAT were observed on portions of the dendritic plasma membrane that were either distant from recognizable synaptic junctions (Fig. $1 A-C$ ) or lateral to the postsynaptic densities (Fig. $1 A, C$ ). In some of the dendrites that received multiple afferent inputs, DAT immunola- 

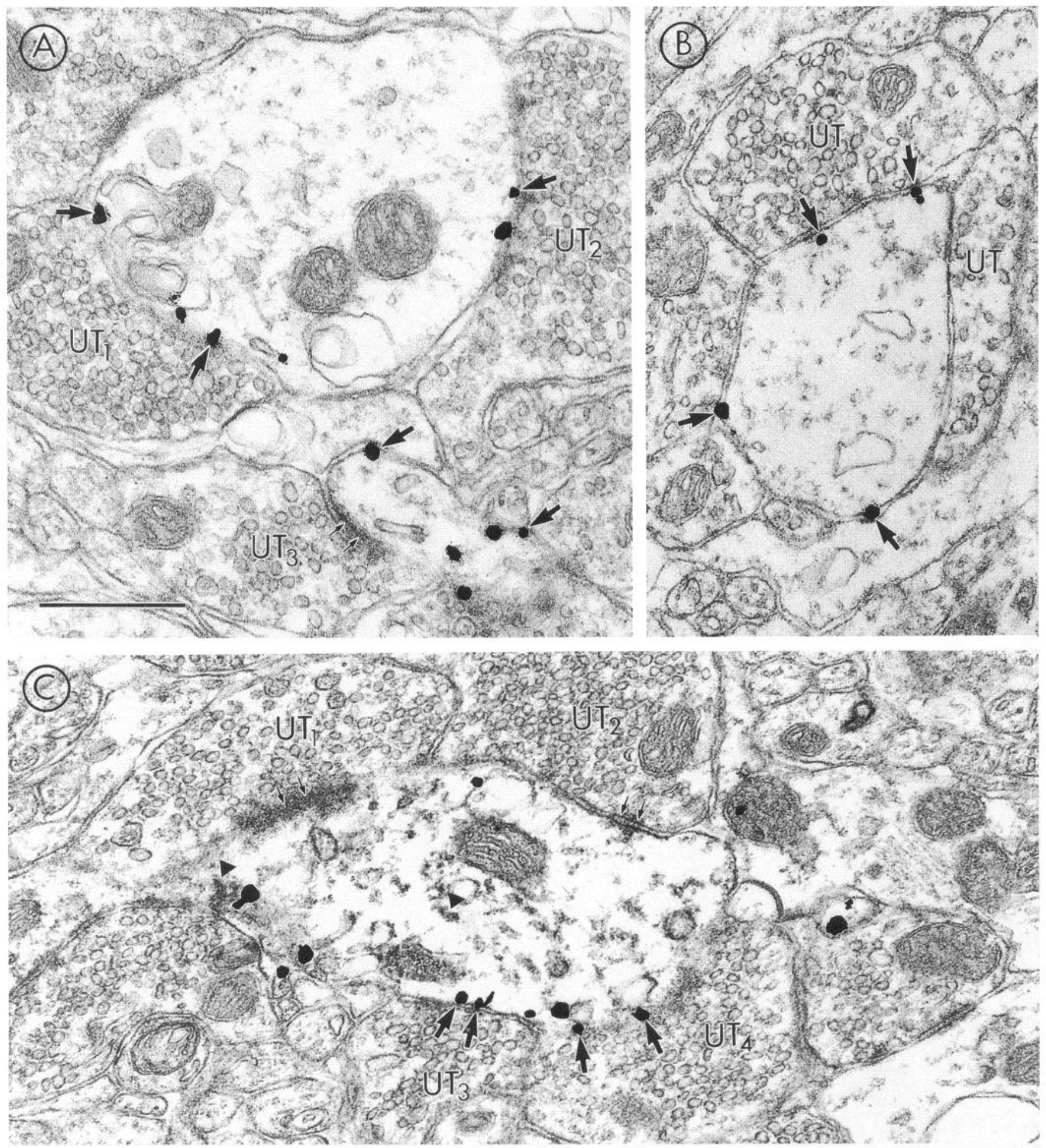

Figure 1. Immunogold labeling for DAT is expressed prominently along plasma membranes of TH-immunoreactive dendrites in the SN. Electron micrographs show immunogold-silver particles for DAT (large arrows) contacting cytoplasmic surfaces of dendritic plasma membranes in the SNR. $A, B$, Tissue was singly immunogold-silver-labeled for DAT. Gold particles are localized prominently to portions of the plasma membrane that contact unlabeled terminals $\left(U T_{1}\right.$ and $U T_{2}$ in $A ; U T$ in $B$ ) but are not detected near a typical asymmetric synaptic junction (small arrows) formed by an unlabeled terminal $\left(U T_{3}\right.$ in $\left.A\right)$. C, Tissue was immunogold-labeled for DAT and immunoperoxidase-labeled (arrowheads) for TH. A large dendrite contains both numerous immunogold particles for DAT and diffuse immunoperoxidase labeling for TH. Two unlabeled terminals $\left(U T_{1}, U T_{2}\right)$ form synaptic junctions (small arrows) onto portions of the dendrite devoid of immunogold labeling for DAT. Other terminals $\left(U T_{3}, U T_{4}\right)$ contact portions of the dendrite on which several gold particles for DAT are seen. Scale bar, $0.5 \mu \mathrm{m}$. 
beling was localized to segments of the dendritic plasma membrane that were apposed to specific axon terminals, but labeling was not detected on segments of the plasma membrane near other terminals (Fig. 1C).

In addition to the prominent plasmalemmal labeling for DAT, immunogold particles for DAT were also observed along internal membranes in dendrites (Fig. 2). These immunogold particles often were associated selectively with tubulovesicular structures (Fig. $2 A-C$ ), most of which resembled saccules of smooth endoplasmic reticulum (SER) (Peters et al., 1991). None of the DATlabeled dendrites, however, contained labeled or unlabeled aggregates of synaptic-like vesicles.

In sections processed for dual localization of $\mathrm{DAl}$ ' and ' $\mathrm{IH}$, a majority of the labeled dendrites appeared to contain detectable immunoreaction product for both markers. This was obscrved in sections containing immunogold labeling for DAT and immunoperoxidase labeling for TH (Figs. $1 C, 2 B, C, 3 C$ ) and in sections in which the immunocytochemical markers were reversed (Fig. 3A$B$ ). In contrast to the pattern of immunogold labeling for DAT, which was associated selectively with the plasma membrane and tubulovesicular structures, the immunogold labeling for $\mathrm{TH}$ was expressed more diffusely throughout the cyloplasm of the labeled dendrites and showed no apparent association with specific membranes or subcellular organelles (Fig. $3 A, B$ ).

In some of the dually labeled sections in the SNR, the most intense immunoperoxidase labeling (Fig. $3 A, B$ ) and many of the immunogold particles (Fig. $3 C$ ) for DAT were localized to dendritic spines. In immunoperoxidase-labeled spines, the reaction product for DAT was most intense along the plasma membranes but also was distributed diffusely throughout the cytoplasm (Fig. $3 A, B)$. In contrast, immunogold labeling for $\mathrm{D} \Lambda \mathrm{T}$ was localized almost exclusively along cytoplasmic surfaces of the plasma membrane (Fig. $3 C$ ). In comparison with the densely labeled spines, nonspinous portions of these dendrites contained less intense immunoperoxidase labeling (Fig. $3 A, B$ ) and more sparsely distributed immunogold particles (Fig. $3 C$ ) for DAT. The immunogold (Fig. $3 A, B$ ) and immunoperoxidase (Fig. $3 C$ ) labeling for $\mathrm{TH}$, however, was distributed more diffusely throughout the main portion of the dendrite and showed no specific association with dendritic spines.

\section{DAT is associated with cytoplasmic tubulovesicles in soma in the $\mathbf{S N}$}

DAT-immunoreactive perikarya were also frequently observed in the SNC. In some sections, more isolated DAT-immunoreactive perikarya also extended ventrally from the SNC to the SNR. Ultrastructural examination of the DAT-immunoreactive perikarya showed that they contained immunogold particles for DAT (Fig. $4 A, B)$, most of which were localized to pleomorphic electron-lucent tubulovesicles that were distributed throughout the cytoplasm (Fig. $4 A$ ). Some of these immunogold particles were located near the rough endoplasmic reticulum or early Golgi apparatus (Griffiths and Simons, 1986) (Fig. 4B). Immunogold labeling for DAT was detected more rarcly along the plasma membranes of the labeled perikarya (Fig. 4A). Although mitochondria and, occasionally, dense core vesicles could be seen within the cytoplasm of some of the laheled perikarya, these organelles did not contain detectable immunogold labeling for DAT. In sections that were dually labeled with immunogold for DAT and with immunoperoxidase for $\mathrm{TH}$ (data not shown), most of the labeled perikarya contained detectable immunoredction product for both antigens. This was also the case when the immunocytochemical markers were reversed (data not shown).

\section{DAT localizes to the plasma membranes of varicose axons and axon terminals in the dorsolateral striatum}

In the dorsolateral striatum, the immunogold labeling for DAT was localized to unmyelinated varicose axons (Fig. $5 A, B$ ) and axon terminals (Figs. $5 C, D, 6,7$ ). The DAT-immunoreactive axons were characterized by boutons en passant (Peters et al., 1991), which were filled with aggregates of electron-lucent vesicles, and narrow intervaricose segments that contained fewer, more sparsely distributed vesicles (Fig. $5 A, B$ ). When these axons were observed in a longitudinal plane of scetion, the immunogold labeling for DAT appeared to be associated most prominently with the plasma membranes of houtons en passant (Fig. $5 A, B$ ) but also was seen along plasma membranes of intervaricose segments (Fig. 5A). Within the boutons en passant, the immunogold labeling for DAT appeared to be localized selectively to portions of the plasma membrane located near clusters of electron-lucent vesicles (Fig. 5B) and was detected less frequently along portions of the plasma membrane that were near microtubules. Immunogold particles for DAT were also localized to pleomorphic vesicles, typically in varicose axons in which there was more prominent plasmalemmal labeling for DAT (Fig. 544).

DAT was also localized extensively to axon terminals (boutons terminaux) (Peters et al., 1991) in the dorsolateral striatum (Figs. $5 C, D, 6,7)$. Within these axon terminals, most of the immunogold particles (Figs. $5 C, D, 6 B-E, 7$ ) and intense immunoperoxidase labeling (Fig. $6 A$ ) for DAT appeared to be localized to cytoplasmic surfaces of the plasma membranes. Immunoperoxidaselabeled axon terminals also contained diffuse peroxidase reaction product for DAT, including prominent immunolabeling of both small clectron-lucent vesicles and larger SER tubulovesicles (Fig. $6 A$ ). In immunogold-labeled sections, DAT also was localized more rarely to small electron-lucent vesicles (Fig. 7C). These labeled vesicles usually were detected in axon terminals in which there was also more prominent labeling for DAT along the plasma membrane. The DAT-immunoreactive terminals did not contain any detectable labeled or unlabeled dense-core vesicles.

The DAT-immunoreactive axon terminals in the dorsolateral striatum frequently contacted one or more dendritic spines (Fig. $6 A, C-E$ ) and/or small (Fig. $6 B, D, E$ ) or large (Fig. 7) unlabeled dendrites. These contacts were characterized by parallel, apposed plasma membranes with postsynaptic densities that either were poorly defined (Fig. 7) or, if present, could not be detected (Fig. 6). The plasmalemmal immunogold labeling for DAT was seen both near (Fig. 7) and distant from (Fig. 6) these sites of contact, but rarely over the contacts themselves. In some cases the labeled segments of plasma membrane were apposed to unlabeled dendrites or spines (Fig. 6), whereas in other cases they were detected along nonsynaptic portions of the plasma membrane of the presynaptic axon or axon terminal (Fig. $7 A$ ).

In sections that were double-labeled for DAT and TH, most of the DAT-containing axons and axon terminals in the striatum also contained immunoreaction product for TH. This was seen both in sections that were immunogold-labeled for DAT and immunoperoxidase-labeled for TH (Figs. $5 C, D, 6 C-E, 7$ ), as well as in sections in which the markers were reversed. In contrast to the selective immunogold labeling for DAT, both immunoperoxidase and immunogold reaction products for $\mathrm{TH}$ were distributed diffusely throughout the cytoplasm of the labeled axons and showed no apparent association with specific subcellular organelles. 


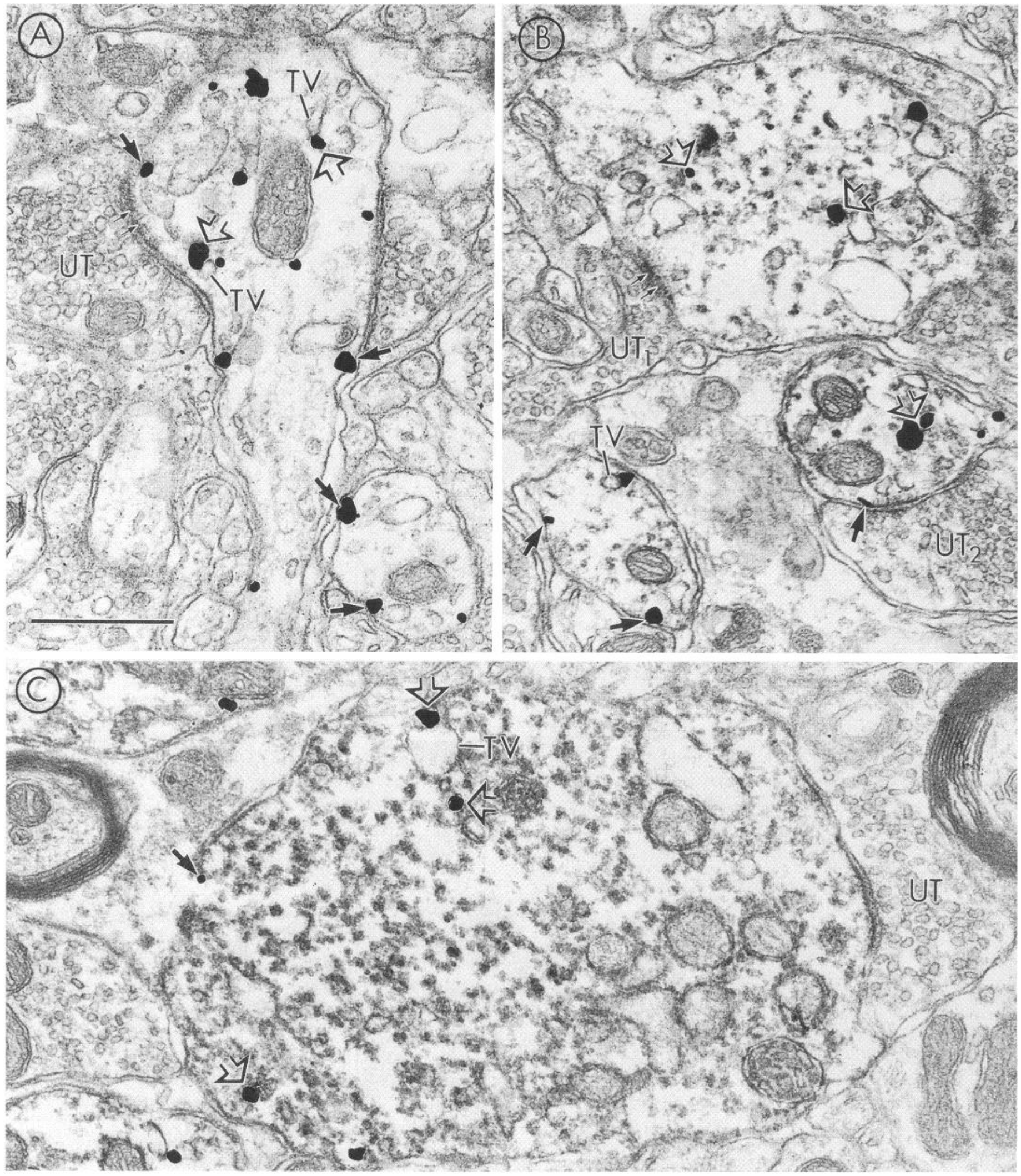

Figure 2. DAT is localized to the SER and plasma membranes of TH-immunoreactive dendrites in the SN. Electron micrographs show immunogold particles for DAT along the plasma membranes (large closed arrows) and internal membranes (open arrows) of dendrites. A, Tissue has been singly immunogold-silverlabeled for DAT. Gold particles are localized to the plasma membranes of two dendrites in the SNR. The larger dendrite also contains extensive immunogold labeling for DAT along tubulovesicles (TV) of SER. An unlabeled terminal $(U T)$ forms a synaptic junction onto the larger DAT-immunoreactive dendrite. $B$, $C$, Sections were sampled from the SNC and were double-labeled with immunogold-silver for DAT and with immunoperoxidase for TH. All of the dendrites that are immunogold-labeled for DAT also contain light peroxidase reaction product for TH. The labeled dendrites contain immunogold particles for DAT along tubulovesicles $(T V)$ of the SER and cytoplasmic surfaces of the plasma membrane. Scale bar, $0.5 \mu \mathrm{m}$. 

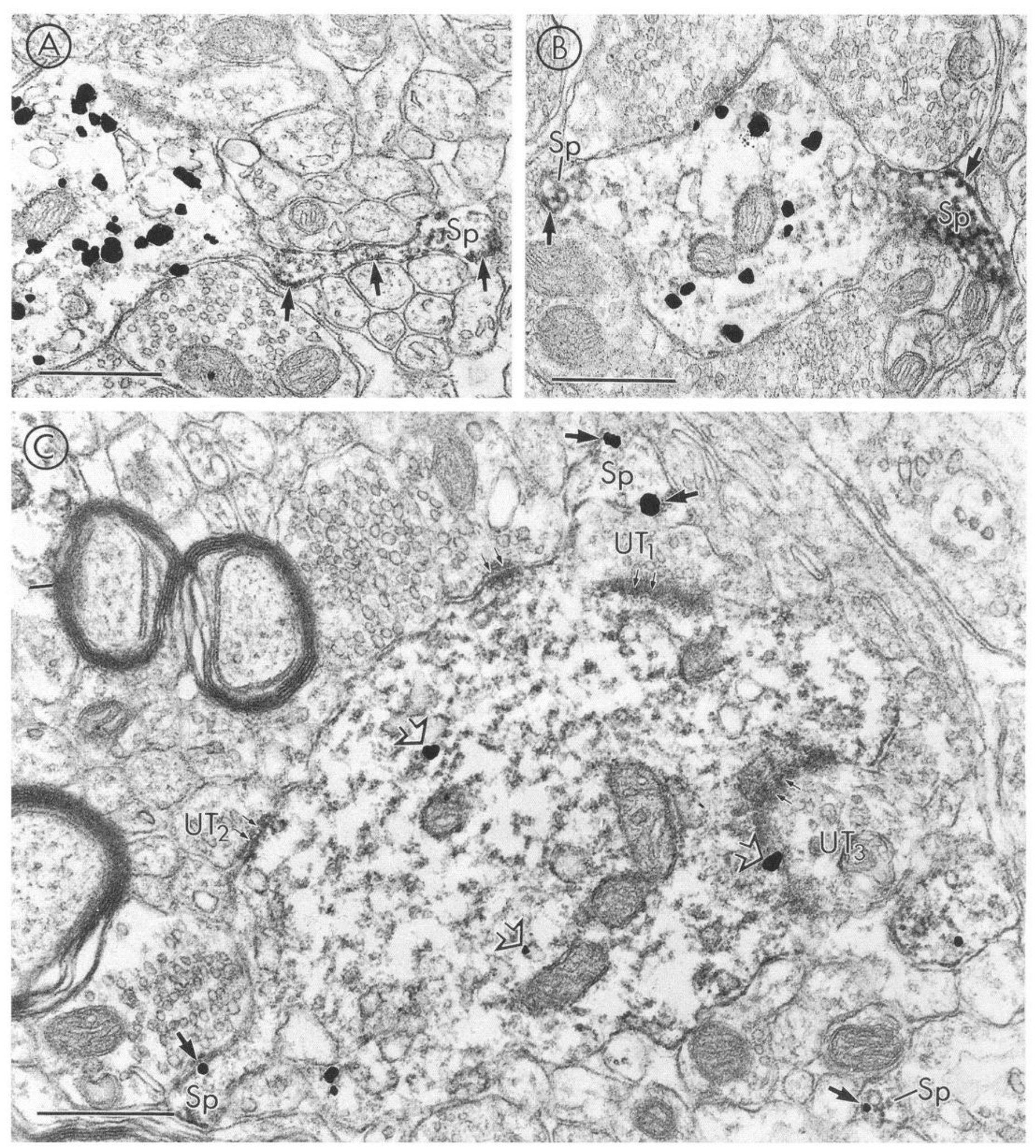

Figure 3. DAT is intensely localized to dendritic spines of dopaminergic neurons in the SNR. Electron micrographs show sections that were double-labeled for DAT and for the catecholamine-synthesizing enzyme TH. $A, B$, Intense immunoperoxidase labeling for DAT is seen along the plasma membranes (large closed arrows) of the head and neck of the spines ( $S p)$ of dendrites, which also contain numerous immunogold particles for TH. $C$, The immunocytochemical markers have been reversed. Immunogold particles for DAT (large arrows) are localized to the plasma membranes of the spines ( $S p$ ) of a large dendrite that also contains diffuse peroxidase reaction product for TH. Immunogold labeling for DAT also is seen along internal membranes (open arrows) of the labeled dendrite. The labeled dendrite receives synaptic contacts (small closed arrows) from unlabeled terminals $\left(U T_{1}, U T_{2}, U T_{3}\right)$. An immunogold particle for DAT (large arrow) is located near an obliquely sectioned synaptic junction (small arrows) formed by one of these unlabeled terminals $\left(U T_{3}\right)$. Small arrows are synaptic junctions. Scale bars, $0.5 \mu \mathrm{m}$. 


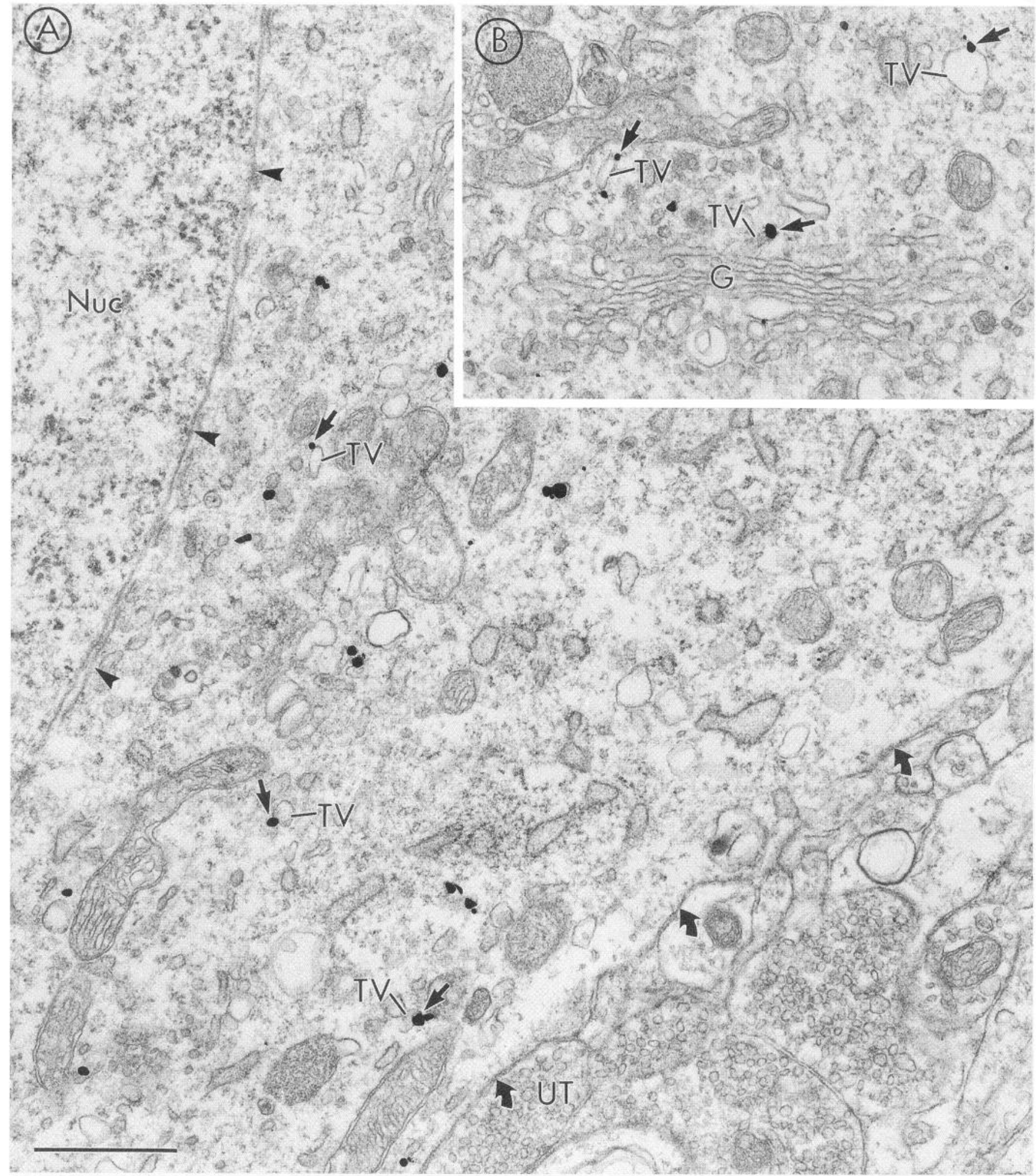

Figure 4. Immunogold labeling for DAT is localized to internal membranes of neuronal perikarya in the SNC. Electron micrographs show immunogold particles for DAT (straight arrows) that are associated with pleomorphic tubulovesicular membranes (TV) in the cytoplasm of two neuronal perikarya. $A$, Immunogold particles are seen throughout the cytoplasm but are not associated with the plasma membrane (curved arrows) or nuclear membrane (arrowheads). B, Immunogold particles for DAT are localized to tubulovesicular membranes (TV) near the Golgi apparatus (G). Nuc, nucleus; UT, unlabeled terminal. Scale bar, $0.5 \mu \mathrm{m}$. 

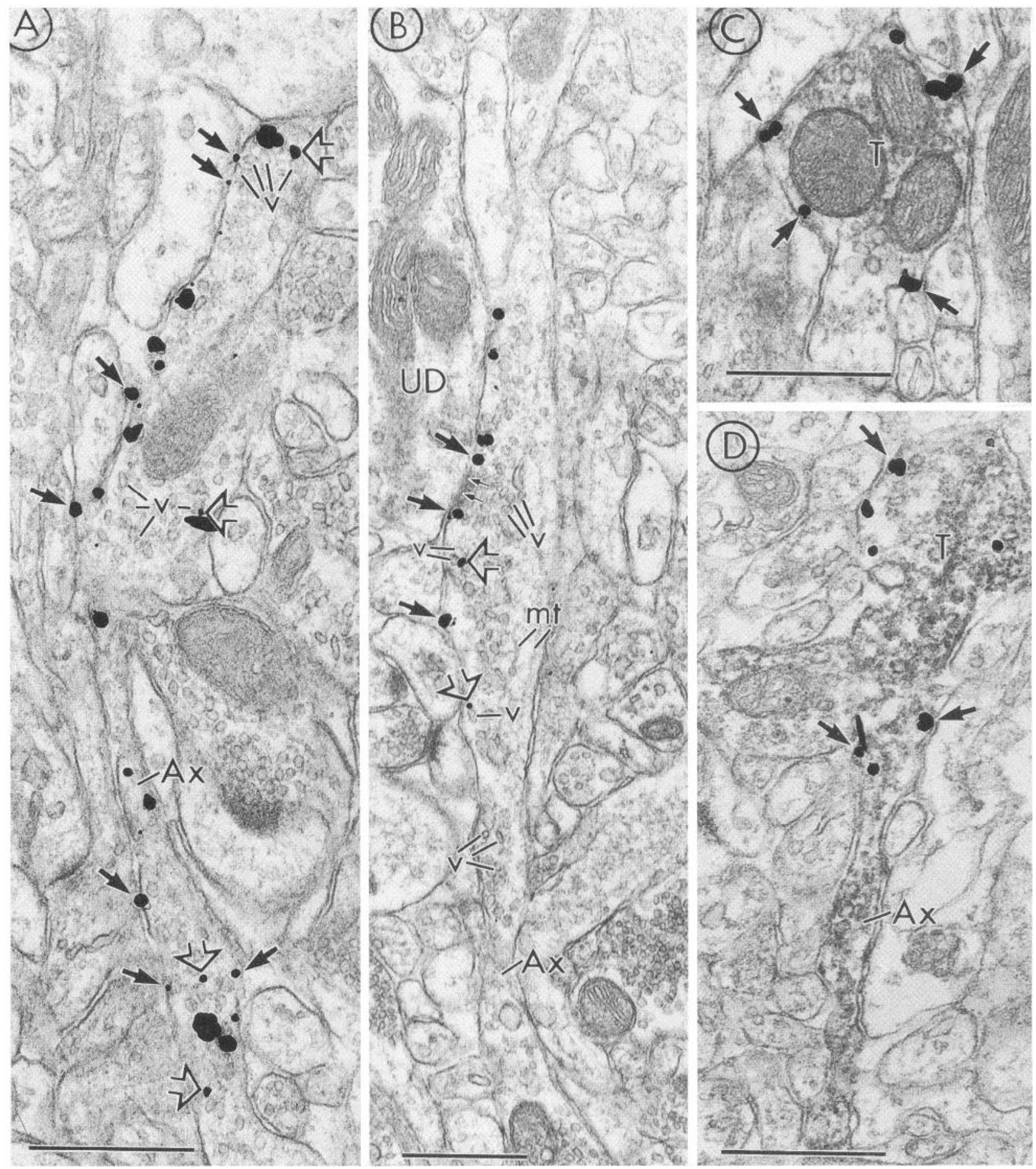

Figure 5. Immunogold labeling for DAT is localized to plasma membranes and pleomorphic tubulovesicles in TH-immunoreactive varicose axons in the dorsolateral striatum. Electron micrographs show immunogold-silver labeling for DAT in unmyelinated axons and axon terminals. $A$, Tissue has been single-labeled for DAT. Numerous immunogold particles for DAT (large closed arrows) directly contact the plasma membrane of an unmyelinated axon $(A x)$. Immunogold particles for DAT are most numerous in a varicose portion of the axon (bouton en passant), where they are located near aggregates of electron-lucent vesicles (v), some of which are also immunogold-labeled for DAT (open arrows). In intervaricose portions of the labeled axons, immunogold particles for DAT also are seen along the plasma membrane (large closed arrows) but are associated more frequently (open arrows) with pleomorphic electron-lucent vesicles $(v) . B$. Immunogold labeling for DAT is seen along the plasma membrane of a bouton en passant that appears to form a synaptic junction (small closed arrows) onto an adjacent unlabeled dendrite $(U D)$. The immunogold particles for DAT are located selectively along portions of the plasma membrane near clusters of unlabeled vesicles $(v) . C, D$, Sections also have been immunoperoxidase-labeled for TH. Immunogold labeling for DAT (large closed arrows) is localized to the plasma membranes of axon terminals $(T)$, which also contain diffuse immunoperoxidase labeling for TH. The preterminal portion of the axon in $D$ also contains intense immunoperoxidase labeling for TH but few immunogold particles for DAT. $m t$, Microtubules. Scale bars, $0.5 \mu \mathrm{m}$. 


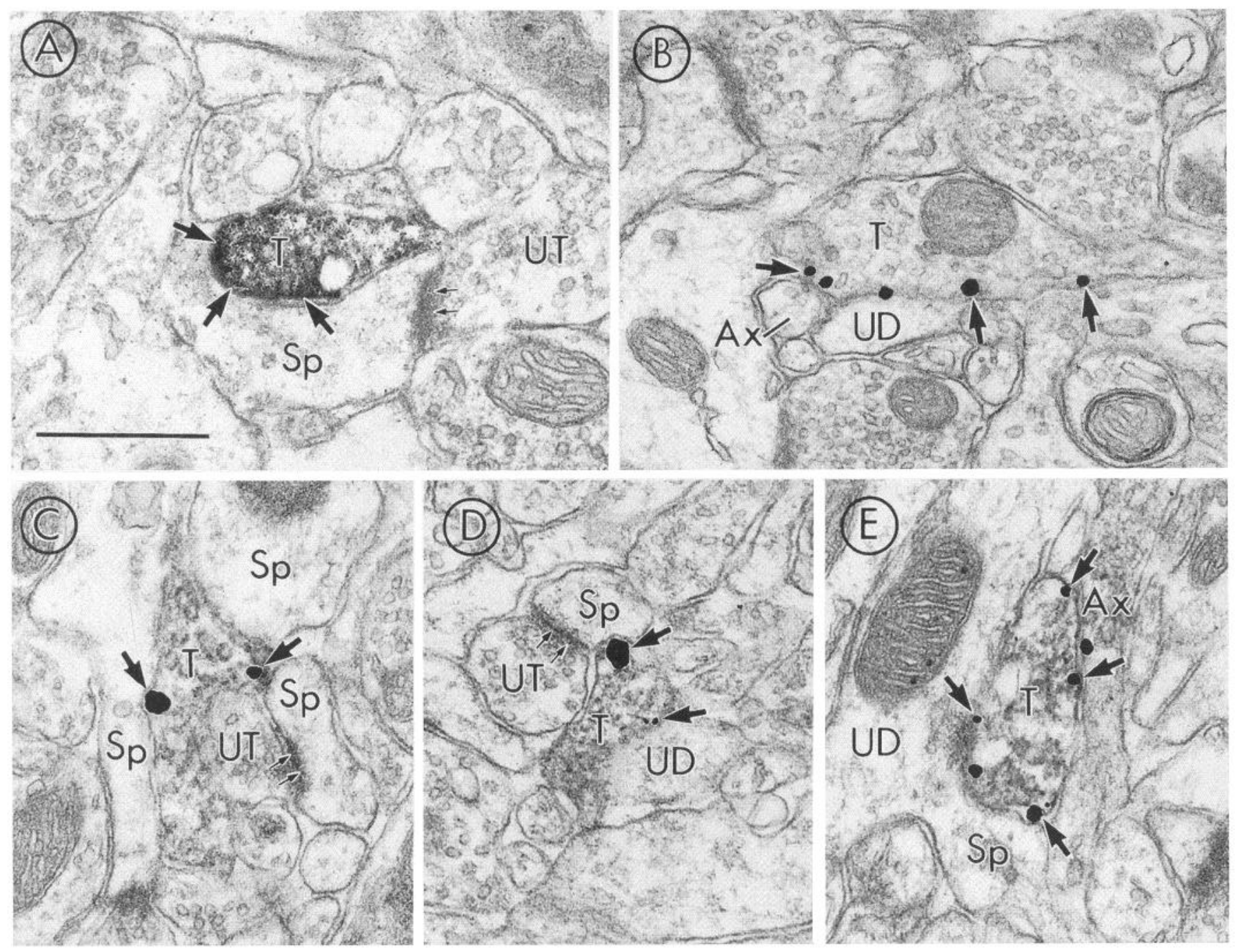

Figure 6. DAT is intensely localized to the plasma membranes of TH-immunoreactive axon terminals in the dorsolateral striatum, many of which are apposed to small dendrites or dendritic spines. $A$, Intense immunoperoxidase labeling for DAT is seen along the plasma membrane (large arrows) of an axon terminal $(T)$ where it contacts an unlabeled dendritic spine $(S p)$. B, Immunogold particles for DAT are localized to the plasma membrane of an axon terminal at its points of contact with a small, unlabeled dendrite $(U D)$ and an unmyelinated axon $(A x)$. $C-E$, Tissue has been double-labeled with immunogold-silver for DAT and with immunoperoxidase for TH. Immunogold particles for DAT (large arrows) are seen along the plasma membranes of axon terminals in which there is also light immunoperoxidase reaction product for $\mathrm{TH}$. These immunogold particles frequently are seen along portions of the plasma membrane that contact unlabeled dendrites $(U D)$ or dendritic spines $(S p)$. Some of the unlabeled spines that are contacted by DAT-labeled terminals also receive asymmetric synapses (small arrows in $A, C$, and $D$ ) from unlabeled terminals (UT). Ax, unlabeled axon. Scale bar, $0.5 \mu \mathrm{m}$.

\section{DISCUSSION}

The present study shows that DAT preferentially localizes to the plasma membranes of TH-immunoreactive dopaminergic dendrites in the SN and varicose axons in the dorsolateral striatum. This localization supports a role for DAT in reuptake of dopamine into presynaptic dendrites and axon terminals and indicates the potential sites of DAT-mediated dopamine release. These findings also suggest that the DAT-mediated uptake of neurotoxins into nigrostriatal dopaminergic neurons occurs primarily through their distal dendritic and axonal processes.

\section{Methodology}

We examined the electron microscopic localization of DAT using a potent and highly selective N-terminal domain anti-peptide antiserum that has been characterized extensively (Freed et al., 1995; Revay et al., in press). The DAT immunolabeling was highly reproducible and was adsorbed by the peptide against which the antiserum was raised but not by an unrelated DAT peptide. The immunogold labeling for DAT was associated with the cytoplasmic surfaces of membranes, supporting the proposed topological model for DAT that predicts that the N-terminal domain resides within the cytoplasm (Giros et al., 1991; Kilty et al., 1991; Shimada et al., 1991).

The distribution, morphology, and synaptic relationships of DATimmunoreactive neurons were consistent with the known distribution of nigrostriatal dopaminergic neurons, as identified previously by radioactive substrate uptake (Descarries et al., 1980) and with antisera against dopamine (Tuffet et al., 1987) or TH (Pickel, 1986; Maley et al., 1990). Most of the DAT-immunoreactive perikarya and processes also contained detectable immunoreaction product for TH. Because dopamine is the only catecholamine present in perikarya in the SN and is the major catecholamine in terminals of the dorsolateral striatum (Pickel and Sesack, 1995), these observations suggest that DAT is localized primarily or exclusively to dopaminergic neurons in these regions. The immunocytochemical localization of DAT confirms the regional distribution of dopamine 

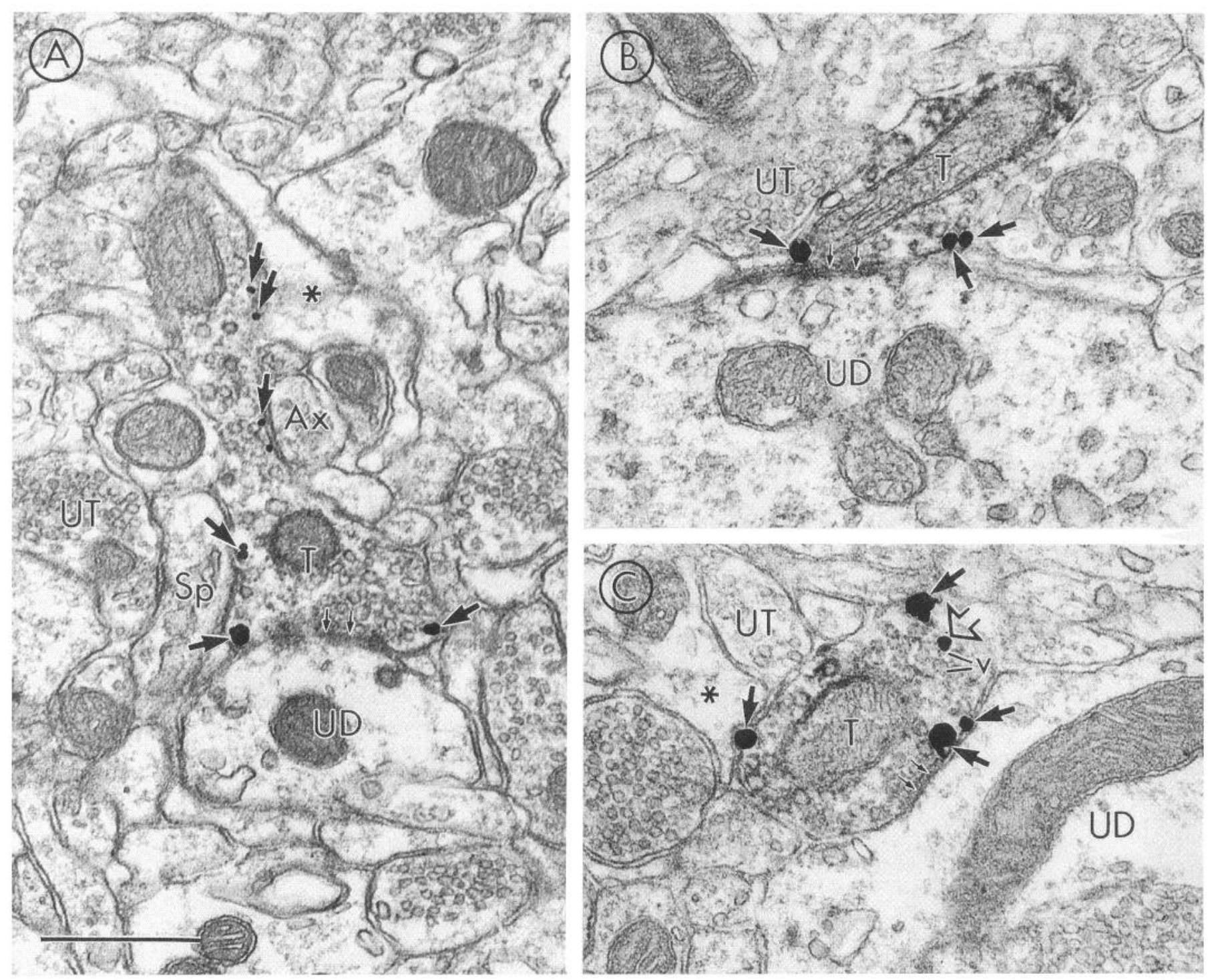

Figure 7. DAT is localized to plasma membranes of TH-immunoreactive axon terminals in the dorsolateral striatum that contact large dendrites. Electron micrographs show sections that have been double-labeled with immunogold for DAT and with immunoperoxidase for TH. Immunogold labeling for DAT (large arrows) is seen along the plasma membranes of axon terminals $(T)$, which also contain light immunoperoxidase labeling for TH. The immunoperoxidase labeling can be appreciated by comparing the electron density of the labeled axon terminals with those of neighboring unlabeled terminals $(U T)$. $A-C$, The labeled terminals $(T)$ contact large, unlabeled dendrites $(U D)$. The sites of contact are evident by the density of the membranes (small arrows) but cannot be defined clearly. The immunogold particles for DAT often are seen lateral to these sites of contact. Immunogold labeling for DAT also is seen on portions of the axonal plasma membrane that are apposed to astrocytes (asterisks) or unlabeled terminals (UT). Scale bar, $0.5 \mu \mathrm{m}$.

uptake sites as identified by radioactive ligand-binding studies (Graybiel and Moratalla, 1989; Cline et al., 1992). The higher resolution of the immunocytochemical techniques, however, has enabled us for the first time to elucidate the precise subcellular localization of DAT in nigrostriatal dopaminergic neurons.

\section{Plasma membranes of nigral dendrites: potential sites of reuptake or dendritic release of dopamine}

DAT was localized prominently to plasma membranes of THimmunoreactive dendrites in the $\mathrm{SN}$, suggesting a role for DAT in dendritic dopamine reuptake. Because we did not detect dopaminergic axon terminals in this region, the dopamine is presumably derived from another source, perhaps the dendrites themselves. This is supported by numerous in vitro and in vivo studies that have shown that dopamine can be released from dendrites in the $\mathrm{SN}$ (Geffen et al., 1976a,b; Cheramy et al., 1981; Bernardini et al., 1991;
Robertson et al., 1991; Rosales et al., 1994). Most of the nigral dendrites in which we observed plasmalemmal labeling for DAT, however, did not contain aggregates of synaptic-like vesicles. In addition, in some cases the most intense labeling for DAT was observed along dendritic spines, which rarely contain synaptic vesicles (Peters et al., 1991). These findings suggest that DAT-mediated dopamine reuptake occurs at sites distant from that of vesiclemediated release. This may result from the diffusion of dopamine through the neuropil (Maley et al., 1990) but also warrants consideration of other possible modes of dopamine release.

The presence of labeling for DAT along segments of dendritic plasma membrane that were distant from synaptic vesicles supports a role for DAT in vesicle-independent dopamine release. Under certain conditions, it has been shown that there is calciumindependent, nonvesicular dopamine release from dendrites in the 
SN mediated by reverse transport of dopamine by DAT (Bannon et al., 1995). This has been demonstrated most convincingly under pharmacological conditions in which the cytosolic concentrations of dopamine are increased, as occurs with reduction of the vesicular $\mathrm{pH}$ gradient by amphetamines or other weak bases (Sulzer et al., 1993; Pifl et al., 1995). There is also evidence that physiological conditions in which there are increased cytoplasmic sodium concentrations, such as glucose deprivation, hypoxia, or the release of excitatory amino acid receptor agonists, can promote reverse transport of dopamine by DAT (Eshleman et al., 1994; Sitges et al., 1994; Bannon et al., 1995). Thus, the presence of DAT along the plasma membranes of dendrites in the SN suggests potential sites for DATmediated dopamine release.

\section{Plasma membranes of striatal terminals: potential sites of DAT-mediated dopamine reuptake or release}

In the striatum, DAT was localized to the plasma membranes of axonal varicosities and axon terminals that contained aggregates of typical synaptic vesicles. This plasmalemmal labeling was observed both near and distant from recognized synaptic junctions. The presence of DAT along segments of the plasma membrane near synaptic specializations supports a role for DAT in regulating the extracellular concentration of dopamine and, thus, in the termination of dopaminergic neurotransmission. Similarly, the localization of DAT to nonsynaptic portions of the plasma membrane may indicate sites at which this transporter regulates the extracellular concentrations of dopamine that has been released from sites lacking well defined synaptic specializations (Beaudet and Descarries, 1978; Maley et al., 1990; Pickel and Sesack, 1995). The frequency with which DAT was observed at nonsynaptic sites also may indicate a role for DAT in the reuptake of dopamine that has diffused through the neuropil and, thus, in the "spatial buffering" of this transmitter (Nicholson and Rice, 1991).

The presence of DAT along the plasma membrane of axon terminals also indicates potential sites for reverse transport and, thus, for calcium- and vesicle-independent release of dopamine in the striatum. As in the $\mathrm{SN}$, reverse transport of dopamine by DAT has been demonstrated most clearly in dopaminergic terminals after application of amphetamines, which increase cytoplasmic concentrations of dopamine (see above). Conceivably, reverse transport of dopamine by DAT also may occur under physiological conditions, including alterations in synaptic inputs, as suggested by studies using pharmacological manipulations that mimic these conditions (Ratieri et al., 1979; Lonart and Zigmond, 1991; Jacocks and Cox, 1992).

\section{Tubulovesicles: possible sites of synthesis, transport, or recycling of DAT}

The DAT-labeled tubulovesicles that were observed throughout neuronal perikarya and processes may represent sites of synthesis, transport, and/or recycling of DAT-containing membranes. Like other plasmalemmal proteins, DAT is believed to be synthesized constitutively in the cell body and subsequently transported to and inserted into the plasma membrane (Bradbury and Bridges, 1994). We have observed extensive immunogold labeling for DAT in pleomorphic tubulovesicles in neuronal cell bodies but little detectable labeling along the perikaryal plasma membrane. This suggests that DAT is synthesized constitutively in the soma but that its primary site(s) of function is in other portions of the cell.

Some of the labeled tubulovesicles in axons and dendrites, therefore, may represent DAT-containing membranes in transit between the cell body and the axonal or dendritic plasma mem- brane. This intracellular pool of DAT may include membranes that have not fused yet with the plasma membrane, and/or membranes that have been recycled from the cell surface (Parton et al., 1992; Bradbury and Bridges, 1994). In addition, many of these DAT-containing tubulovesicles resembled saccules of SER. Interestingly, there is evidence that the SER can sequester monoamines (Mercer et al., 1979; Quatacker et al., 1992; Nirenberg et al., 1995 ) and that the SER may represent the major site of dopamine storage in dendrites (Cheramy et al., 1981). If this is the case, then DAT also may play a role in reducing the lumenal concentrations of dopamine and/or related neurotoxins within saccules of SER.

\section{Implications for dopamine neurotoxicity and idiopathic Parkinson's disease}

Recent studies have shown that DAT is the site of uptake of neurotoxins such as 1-methyl-4-phenylpyridine $\left(\mathrm{MPP}^{+}\right)$and 6-hydroxydopamine (Javitch et al., 1985; Sundstrom et al., 1986; Kitayama et al., 1992; Pifl et al., 1993) into dopaminergic neurons. These neurotoxins cause selective degeneration of dopaminergic neurons and associated symptoms of parkinsonism and, therefore, have been used as models of Parkinson's disease (Kaakkola and Teräväinen, 1990). DAT also may confer selective vulnerability to idiopathic Parkinson's disease, in which it has been suggested that an endogenous neurotoxin leads to preferential degeneration of nigrostriatal dopaminergic neurons (Edwards, 1993; Ben-Shachar et al., 1995).

In this context, the electron microscopic localization of DAT offers additional information regarding the specific subcellular sites at which neurotoxins may enter dopaminergic neurons. Because DAT is expressed at higher levels in the striatum as compared with the $\mathrm{SN}$, the extensive localization of DAT along plasma membranes of striatal axon terminals probably reflects the principal sites through which dopamine neurotoxins enter the cell. The additional prominent labcling for DAT along dendritic plasma membrancs in the SN, most notably membranes of dendritic spines, suggests that there is also considerable uptake of neurotoxins through distal dendrites. Dopaminergic perikarya, however, had almost no detectable immunolabeling along the plasma membrane, suggesting that there is limited uptake of dopamine neurotoxins into the cell body. Together, these findings suggest that dopamine neurotoxins gain entry primarily through the distal axonal and dendritic processes of nigrostriatal dopaminergic neurons.

\section{REFERENCES}

Bannon MJ, Granneman JG, Kapatos G (1995) The dopamine transporter: potential involvement in neuropsychiatric disorders. In: Psychopharmacology: the fourth generation of progress (Bloom FE, Kupfer DJ, eds), pp 179-188. New York: Raven.

Beaudet A, Descarries L (1978) The monoamine innervation of rat cerebral cortex: synaptic and non-synaptic axon terminals. Neuroscience 3:851-860.

Ben-Shachar D, Zuk R, Glinka Y (1995) Dopamine neurotoxicity: inhibition of mitochondrial respiration. J Neurochem 64:718-723.

Bernardini GL, Gu X, Viscardi E, German DC (1991) Amphetamineinduced and spontaneous release of dopamine from A9 and A10 dendrites: an in vitro electrophysiological study in the mouse. $J$ Neural Transm Gen Sect 84:183-193.

Björklund A, Lindvall O (1984) Dopamine-containing systems in the central nervous system. In: Handbook of chemical neuroanatomy, Vol II, Classical transmitters in the central nervous system (Björklund A, Hökfelt T, eds), pp 55-121. Amsterdam: Elsevier Science.

Bradbury NA, Bridges RJ (1994) Rolc of membranc trafficking in plasma membrane solute transport. Am J Physiol 267:C1-C24.

Cerruti C, Walther DM, Kuhar MJ, Uhl GR (1993) Dopamine transporter mRNA expression is intense in rat midbrain neurons and modest outside midbrain. Mol Brain Res 18:181-186. 
Chan J, Aoki C, Pickel VM (1990) Optimization of differential immunogold-silver and peroxidase labeling with maintenance of ultrastructure in brain sections before plastic embedding. J Neurosci Methods 33:113-127.

Cheramy A, Leviel V, Glowinski J (1981) Dendritic release of dopamine in the substantia nigra. Nature 289:537-542.

Ciliax BJ, Heilman C, Demchyshyn LL, Pristupa ZB, Ince E, Hersch SM, Niznik HB, Levey AI (1995) The dopamine transporter: immunocytochemical characterization and localization in brain. $\mathrm{J}$ Neurosci 15:1714-1723.

Cline EJ, Scheffel U, Boja JW, Mitchell WM, Carroll FI, Abraham P, Lewin AH, Kuhar MJ (1992) In vivo binding of $\left[{ }^{125} \mathrm{I}\right] \mathrm{RTI}-55$ to dopamine transporters: pharmacology and regional distribution with autoradiography. Synapse 12:37-46.

Descarries L, Bosler O, Berthelet F, Des Rosiers MH (1980) Dopaminergic nerve endings visualised by high-resolution autoradiography in adult rat neostriatum. Nature 284:620-622.

Edwards RH (1993) Neuronal degeneration and the transport of neurotransmitters. Ann Neurol 34:638-645.

Eshleman AJ, Henningsen RA, Neve KA, Janowsky A (1994) Release of dopamine via the human transporter. Mol Pharmacol 45:312-316.

Freed C, Revay R, Vaughan RA, Kriek E, Grant S, Uhl GR, Kuhar MJ (1995) Dopamine transporter immunoreactivity in rat brain. J Comp Neurol 359:340-349.

Geffen LB, Jessell TM, Cuello AC, Iverson LL (1976a) Release of dopamine from dendrites in rat substantia nigra. Nature 260:258-260.

Geffen LB, Jessell TM, Cuello AC, Iverson LL (1976b) Nigral and striatal dopamine release under sensory stimuli. Nature 269:340-342.

Gerfen CR, Herkenham M, Thibault J (1987) The neostriatal mosaic. II. Patch- and matrix-directed mesostriatal dopaminergic and nondopaminergic systems. J Neurosci 7:3915-3934.

Giros B, El Mestikawy S, Bertrand L, Caron G (1991) Cloning and functional characterization of a cocaine-sensitive dopamine transporter. FEBS Lett 295:149-154.

Graybiel AM, Moratalla R (1989) Dopamine uptake sites in the striatum are distributed differentially in striosome and matrix compartments. Proc Natl Acad Sci USA 86:9020-9024

Griffiths G, Simons K (1986) The trans-Golgi network: sorting at the exit site of the Golgi complex. Science 234:438-443.

Hsu SM, Raine L, Fanger H (1981) The use of avidin-biotin-peroxidase complex $(\mathrm{ABC})$ in immunoperoxidase technique: a comparison between $\mathrm{ABC}$ and unlabeled antibody (peroxidase) procedures. J Histochem Cytochem 29:577-599.

Jacocks HMD, Cox BM (1992) Serotonin-stimulated release of $\left[{ }^{3} \mathrm{H}\right] \mathrm{do}-$ pamine via reversal of the dopamine transporter in rat striatum and nucleus accumbens: a comparison with release elicited by potassium, $N$-methyl-D-aspartic acid, glutamic acid, and D-amphetamine. J Pharmacol Exp Ther 262:356-364.

Javitch JA, D'Amato RJ, Strittmatter SM, Snyder SH (1985) Parkinsonisminducing neurotoxin, $N$-methyl-4-phenyl-1,2,3,6-tetrahydropyridine: uptake of the metabolite $N$-methyl-4-phenylpyridine by dopamine neurons explains sclcctive toxicity. Proc Natl Acad Sci USA 82:2173-2177.

Kaakkola S, Teräväinen H (1990) Animal models of parkinsonism. Pharmacol Toxicol 67:95-100.

Kilty JE, Lorang D, Amara SG (1991) Cloning and expression of a cocaine-sensitive rat dopamine transporter. Science 254:578-579.

Kitayama S, Shimada S, Uhl GR (1992) Parkinsonism-inducing neurotoxin $\mathrm{MPP}^{+}$: uptake and toxicity in nonneuronal COS cells expressing dopamine transporter cDNA. Ann Neurol 32:109-111.

Kuhar MJ, Ritz MC, Boja JW (1991) The dopamine hypothesis of the reinforcing properties of cocaine. Trends Neurosci 14:299-302.

Lonart G, Zigmond MJ (1991) High glutamate concentrations evoke $\mathrm{Ca}^{++}$-independent dopamine release from striatal slices: a possible role of reverse dopamine transport. J Pharmacol Exp Ther 256:1132-1138.

Maley BE, Engle MG, Humphries S, Vascik DA, Howes KA, Newton BW, Elde RP (1990) Monoaminc synaptic structurc and localization in the central nervous system. J Electron Microse Tech 15:20-33.

Manaye KF, Sonsalla PK, Barnett G, Heikkila RE, Woodward DJ, Smith WK, German DC (1989) 1-Methyl-4-(2'-methylphenyl)-1,2,3,6-tetrahydropyridine $\left(2{ }^{\prime}-\mathrm{CH}_{3}-\mathrm{MPTP}\right)$-induced degeneration of mesostriatal dopaminergic neurons in the mouse: biochemical and neuroanatomical studies. Brain Res 491:307-315.

Mercer L, del Fiacco M, Cuello AC (1979) The smooth endoplasmic reticulum as a possible storage site for dendritic dopamine in substantia nigra neurones. Experientia 35:101-103.
Nicholson C, Rice ME (1991) Diffusion of ions and transmitters in the brain cell microenvironment. In: Volume transmission in the brain: novel mechanisms for neural transmission (Fuxe K, Agnati LF, eds), pp 279-294. New York: Raven

Nirenberg MJ, Liu Y, Peter D, Edwards RH, Pickel VM (1995) The vesicular monoamine transporter-2 is present in small synaptic vesicles and preferentially localizes to large dense core vesicles in rat solitary tract nuclei. Proc Natl Acad Sci USA 92:8773-8777.

Nissbrandt H, Sundstrom E, Jonsson G, Hjorth S, Carlsson A (1989) Synthesis and release of dopamine in rat brain: comparison between substantia nigra pars compacta, pars reticulata, and striatum. J Neurochem 52:1170-1182.

Parton RG, Simons K, Dotti CG (1992) Axonal and dendritic endocytic pathways in cultured neurons. J Cell Biol 119:123-137.

Peters A, Palay SL, Webster HD (1991) The fine structure of the nervous system. New York: Oxford UP.

Pickel VM (1986) Ultrastructure of central catecholaminergic neurons. In: Neurohistochemistry: modern methods and applications (Panula P, Paivarinta H, Soinila S, eds), pp 397-423. New York: Liss.

Pickel VM, Johnson E, Carson M, Chan J (1992) Ultrastructure of spared dopamine terminals in caudate-putamen nuclei of adult rats neonatally treated with intranigral 6-hydroxydopamine. Brain Res 70:75-86.

Pickel VM, Sesack SR (1995) Electron microscopy of central dopamine systems. In: Psychopharmacology: the fourth generation of progress (Bloom FE, Kupfer DJ, eds), pp 257-268. New York: Raven.

Pifl C, Drobny H, Reither H, Hornykiewicz O, Singer EA (1995) Mechanism of the dopamine-releasing actions of amphetamine and cocaine: plasmalemmal dopamine transporter versus vesicular monoamine transporter. Mol Pharmacol 47:368-373

Pifl C, Giros B, Caron MG (1993) Dopamine transporter expression confers cytotoxicity to low doses of the parkinsonism-inducing neurotoxin 1-methyl-4-phenylpyridinium. J Neurosci 13:4246-4253.

Quatacker JR, Annaert WG, Miserez BJ, De Potter WP (1992) Immunocytochemical demonstration of dopamine- $\beta$-hydroxylase and cytochrome B561 on the axonal reticulum in bovine sympathetic neurons. J Histochem Cytochem 40:1599-1604

Ratieri M, Cerrito F, Cervoni AM, Levi G (1979) Dopamine can be released by two mechanisms differentially affected by the dopamine transport inhibitor nomifensine. J Pharmacol Exp Ther 208:195-202.

Revay R, Vaughan R, Grant S, Kuhar MJ (1996) Dopamine transporter immunohistochemistry in median eminence, amygdala and other areas. Synapse, in press.

Ritz MC, Lamb RJ, Goldberg SR, Kuhar MJ (1987) Cocaine receptors on dopamine transporters are related to self-administration of cocaine. Science 237:1219-1223.

Robertson GS, Damsma G, Fibiger HC (1991) Characterization of dopamine release in the substantia nigra by in vivo microdialysis in freely moving rats. J Neurosci 11:2209-2216.

Rosales MG, Flores G, Hernández S, Martínez-Fong D, Aceves J (1994) Activation of subthalamic neurons produces NMDA receptor-mediated dendritic dopamine release in substantia nigra pars reticulata: a microdialysis study in the rat. Brain Res 645:335-337.

Roth RH, Elsworth JD (1995) Biochemical pharmacology of midbrain dopamine neurons. In: Psychopharmacology: the fourth generation of progress (Bloom FE, Kupfer DJ, eds), pp 227-243. New York: Raven.

Seeman P (1995) Dopamine receptors: clinical correlates. In: Psychopharmacology: the fourth generation of progress (Bloom FE, Kupfer DJ, eds), pp 295-302. New York: Raven.

Shimada S, Kitayama S, Lin CL, Patel A, Nanthakumar E, Gregor P, Kuhar M, Uhl G (1991) Cloning and expression of a cocaine-sensitive dopamine transporter complementary DNA [published erratum appears in Science (1992) 255:1195]. Science 254:576-578.

Shimada S, Kitayama S, Walther D, Uhl G (1992) Dopamine transporter mRNA: dense expression in ventral midbrain neurons. Mol Brain Res $13: 359-362$.

Sitges M, Reyes A, Chiu LM (1994) Dopamine transporter mediated release of dopamine: role of chloride. J Neurosci Res 39:11-22.

Sulzer D, Maidment NT, Rayport S (1993) Amphetamine and other weak bases act to promote reverse transport of dopamine in ventral midbrain neurons. J Neurochem 60:527-535.

Sundstrom E, Goldstein M, Jonsson G (1986) Uptake inhibition protects nigro-striatal dopamine neurons from the neurotoxicity of 1-methyl-4phenylpyridine (MPP ${ }^{+}$) in mice. Eur J Pharmacol 131:289-292.

Tuffet S, Grandier-Vazeilles X, Geffard M (1987) Monoclonal antibodies against glutaraldehyde-conjugated dopamine. J Neurochem 49:487-49.4. 